\title{
Effects of brackets and ties on stiffness of an arch wire
}

\author{
David M. Adams, D.D.S., M.S., John M. Powers, Ph.D.,** and Kamal Asgar, Ph.D.** \\ Saginaw and Ann Arbor, Mich.
}

\begin{abstract}
Beam theory was used to evaluate the stiffness of a simulated orthodontic model as affected by the type of bracket, interbracket distance, type of ligature tie, and size of segment. For a given deflection, the model generated greater force (increased stiffness) as the beam constant ( $N$ ) increased. $\mathrm{N}$ increased as interbracket distance increased. Metal ties were as rigid or more rigid than $\mathrm{O}$-rings. Four bracket segments were more rigid than two-bracket segments when tied with o-rings but not metal ligatures. Values of $\mathrm{N}$ of Lewis and narrow twin brackets with metal ties were similar and greater than the $\mathrm{N}$ of wide twin and medium single brackets. Wide twin brackets were more rigid than others with o-rings. (AM J ORTHOD DENTOFAC ORTHOP 1987;91:131-6.)
\end{abstract}

Key words: Stiffness, bracket, mechanics, beam theory

$\mathrm{T}$ individual components including brackets, arch wires, O-ring, and metal ligature ties. With numerous forms of each component presently available, the design of an appliance can be manipulated to facilitate treatment of a given malocclusion.

The geometric similarity between components used by the orthodontist and the structural engineer makes it possible to borrow formulas for loads, deflections, and stresses, and apply them to the miniature structures used intraorally during orthodontic treatment. ${ }^{1,2}$

A beam (Fig. 1, A) often presented in orthodontic textbooks ${ }^{1-3}$ is described by Equation 1:

$$
\mathrm{D}=\frac{\mathrm{L}^{3} \mathrm{~F}}{\mathrm{NEI}}
$$

Equation 1

where $F$ is the force, $L$ is the length of the beam, $D$ is the deflection, $\mathrm{E}$ is the modulus of elasticity, and $\mathrm{I}$ is the moment of inertia. $\mathrm{N}$ is a constant that reflects the stiffness of the bearn and is dependent on the supports. When the ends of a beam are free to pivot at the supports, $\mathrm{N}$ equals 48 . When the ends are fixed rigidly (with no rotation or longitudinal movement permitted), $\mathrm{N}=192$. A major difference between a beam and an isolated segment of an orthodontic appliance (Fig. 1,

Based on a thesis submitted in partial fulfillment of the requirements for the master's degree in the Horace H. Rackham School of Graduate Studies at the University of Michigan, 1985.

*Orthodontist practicing in Saginaw, Mich.

**Professor of Dertistry, Department of Biomaterials, School of Dentistry University of Michigan.
$B$ ) is the nature of the supports. For the clinical situation, the rigidity at the supports or anchor teeth depends on interactions among the brackets, arch wire, and ligature ties.

Force is directly proportional to $\mathrm{N}$ in the beam equation. The force necessary to deflect an arch wire a given amount within its elastic range is equal to the reactive force of the arch wire and causes tooth movement. Because an increase in $\mathrm{N}$ results in a proportional increase in the reactive force of the arch wire, it is important to determine how variation in the components of an orthodontic appliance (brackets, arch wires, and ligature ties) influences $\mathrm{N}$.

The purpose of this study was to investigate the stiffness of a simulated orthodontic model. Beam theory was used to evaluate the effects of type of bracket, interbracket distance, type of ligature tie, and size of the segment on the available force.

\section{MATERIALS AND METHODS}

A model was constructed consisting of a jig that functioned as teeth with brackets, a cage to hold the jig during testing, and a hook to deflect the wire (Figs. 2 and 3). The hook engaged the wire at mid span with a length of contact of $0.54 \mathrm{~mm}$. A bracket was not affixed to the hook. The jig was fashioned to allow for variation in the type of anchor brackets, interbracket distance, type of ligature ties (metal or O-ring), and total number of anchor brackets engaging the arch wire.

Four types of $0.56 \times 0.71 \mathrm{~mm}(0.022 \times 0.028$ inch) nontorqued, nonangulated brackets were tested (Fig. 4, $A$ through $D$ ). They included medium single 


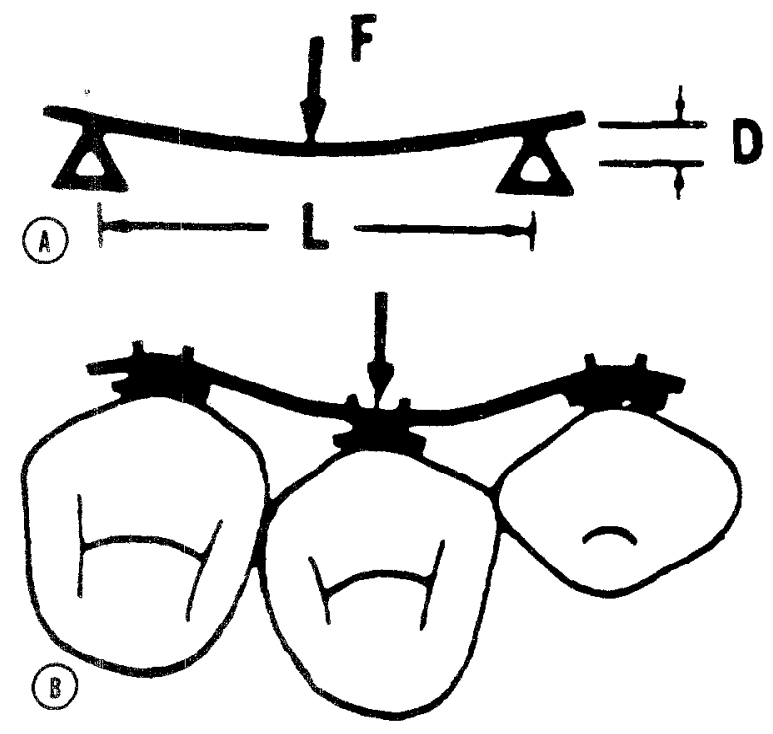

Fig. 1. A, Beam supported at each end (anchor) and loaded in the center. B, Isolated segment of an orthodontic appliance with anchor brackets on either side of activated bracket.

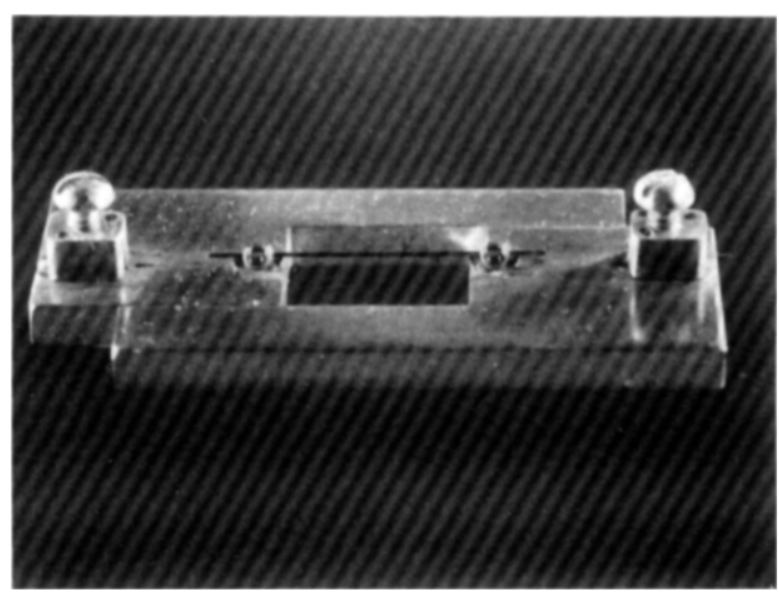

Fig. 2. Jig with anchor brackets and wire in place.

(MS*), narrow twin ( $\left.\mathrm{NT}^{*}\right)$, wide twin (WT $\ddagger$ ), and Lewis ( $L W \S)$ brackets. Intrabracket dimensions were measured to the nearest $0.05 \mathrm{~mm}$.

The interbracket distance $(\mathrm{L})$ represented the length of free wire between the two anchor brackets. Two

*Edgewise bracket, single, $0.018 \times 0.022$ inch slot, batch no. $100-0022$ Ormco Corporation, Glendora, CA 91740.

†Edgewise bracket, small twir, $0.018 \times 0.022$ inch slot, batch no. 100-2022, Ormco Corporation, Glendora, CA 91740.

†Edgewise bracket, large twin, $0.018 \times 0.022$ inch slot, batch no. $100-4022$, Ormco Corporation, Glendora, CA 91740 .

SEdgewise bracket, Lewis, $0.018 \times 0.022$ inch slot, batch no. 115-8822, Onnco Corporation, Glendora, CA 91740 .

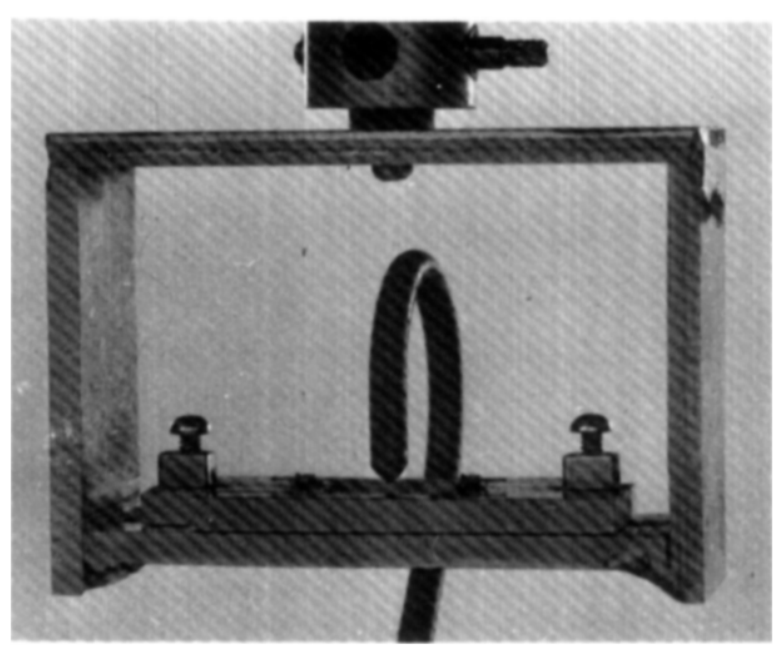

Fig. 3. Jig, cage, and hook in test position. The hook engages the wire at midspan over a length of contact of $0.54 \mathrm{~mm}$.

interbracket distances of $21.25 \mathrm{~mm}\left(\mathrm{~L}_{1}\right)$ and $27.50 \mathrm{~mm}$ $\left(\mathrm{L}_{2}\right)$ measured to the nearest $0.05 \mathrm{~mm}$ were tested.

The $0.457 \mathrm{~mm}(0.018 \mathrm{inch})^{*}$ stainless steel round arch wire was secured to the anchor brackets by one of four methods. For one subsample, the arch wire simply laid within the bracket slots (free) and no ligature ties were placed. In the second subsample, O-rings (E⿱丶万) were used. Metal ligature ties $\ddagger(\mathrm{M})$ were placed in the third subsample. For the final subsample, the arch wire was fixed rigidly to the anchor brackets by filling the bracket slots with composite resin. The resin prevented rotation and longitudinal movement of the wire.

The numbers of anchor brackets engaging the arch wire were varied from two (2b) to four (4b). This simulated testing either a small two-bracket anchor or larger four-bracket anchor of the appliance. The distance between two brackets of the four bracket anchor was $10 \mathrm{~mm}$.

Tests were run with the jig (prepared with a given set of variables), cage, and hook positioned in a testing machine. $\$$ A load was applied and load-deflection measurement recorded at $0.10 \mathrm{~mm}$ increments up to 1.5 $\mathrm{mm}$ of total deflection. The dial that measured deflection on the testing machine was accurate to $0.05 \mathrm{~mm}$.

The modulus of elasticity $(E)$ of the wire was measured in tension on a testing machineß with an exten-

*Orthodontic wire, Tru-chrome, 0.018 inch diameter, batch no. 2593, Rocky Mountain/Orthodontics, Denver, CO 80204.

$\dagger$ Power O's, elastic no. 120, Ormco Corporation, Glendora, CA 91740.

¥Ligature wire, dead soft stainless steel, 0.010 inch diameter, Unitek Corporation, Monrovia, CA 91036.

\$Model TT-BM, Instron Corporation, Canton, MA 02021. 


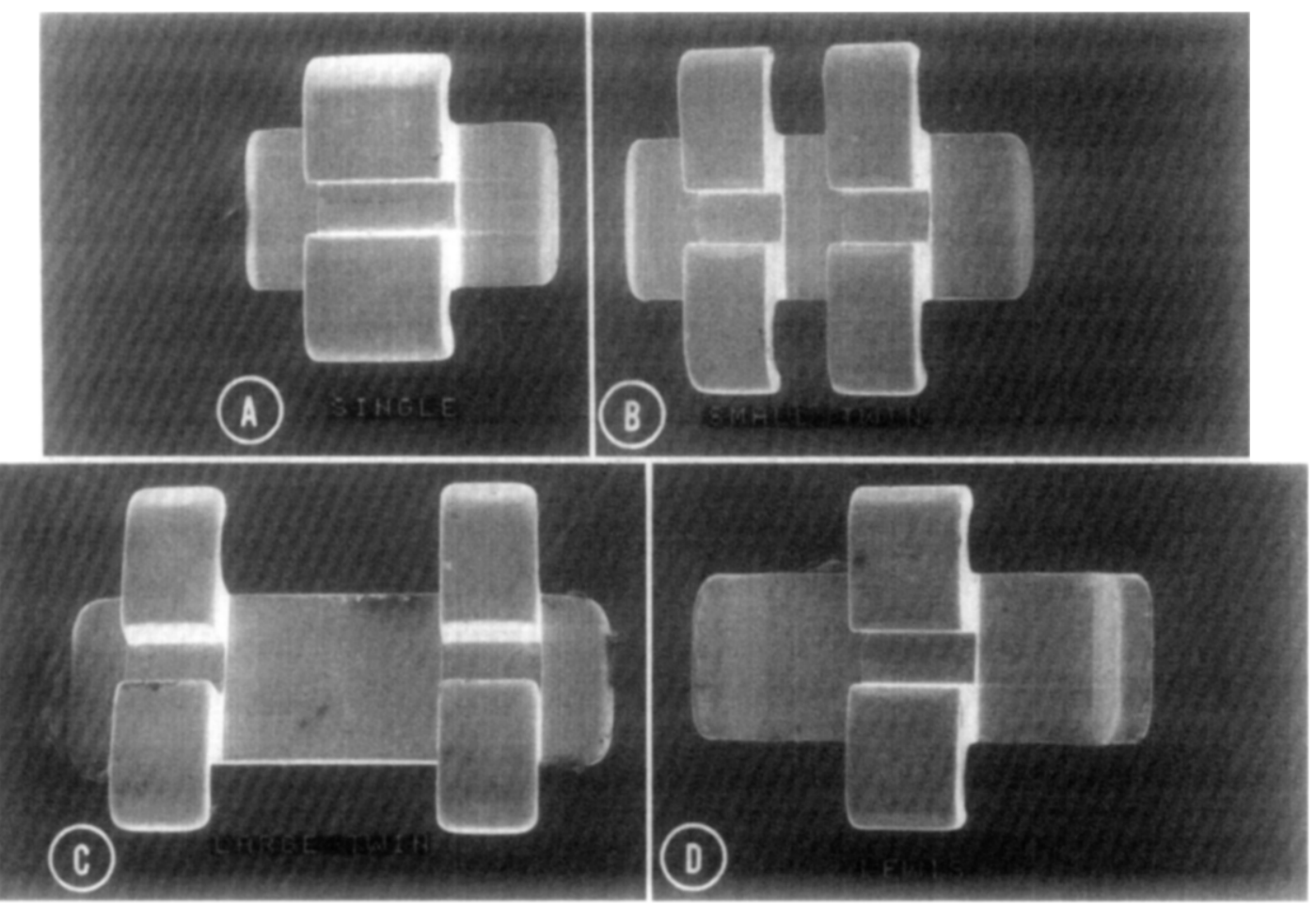

Fig. 4. Four types of brackets tested. A, Medium single. B, Narrow twin. C, Wide twin. D, Lewis.

someter.* The diameters of six as-received $15 \mathrm{~cm}$ lengths of the $0.45 \mathrm{~mm}(0.018 \mathrm{inch})$ stainless steel wire were measured to the nearest $0.05 \mathrm{~mm}$. Tests were run with a crosshead speed of $0.5 \mathrm{~mm}$ per minute. The gauge length was $25.4 \mathrm{~mm}$ ( 1 inch). From load-deflection data, stress-strain curves were determined and the modulus calculated. The moment of inertia (I) was computed using the average wire diameter.

Thirty-two combinations of samples were prepared. Three trials were run for each sample yielding a total of 96 tests. Load-deflection data from each test were plotted and the slope $(\Delta \mathrm{F} / \Delta \mathrm{D})$ of the linear portion determined. Equation 1 was solved for $\mathrm{N}$ using the values determined for the slope, interbracket distance (L), modulus of elasticity (E), and moment of inertia (I) to give Equation 2:

$$
\mathrm{N}=\frac{\mathrm{L}^{3} \Delta \mathrm{F}}{\mathrm{EI} \Delta \mathrm{D}} \text { or } \mathrm{L}^{3} / \mathrm{EI} \times \begin{gathered}
\text { (slope of the load- } \\
\text { deflection curve) }
\end{gathered} \text { Equation } 2
$$

The mean and standard deviation of $\mathrm{N}$ were computcd for cach sample. The data were subjected to analysis of variance using a factorial design. ${ }^{4}$ Means were ranked by a Tukey interval ${ }^{5}$ calculated at the $95 \%$ level of confidence. Differences between two means that

*Model No. 651-11M, Instron Corporation, Canton, MA 02021.
Table I. Intrabracket width

\begin{tabular}{l|c|c}
\hline $\begin{array}{c}\text { Type of } \\
\text { bracket }\end{array}$ & Code & $\begin{array}{c}\text { Widih } \\
(\mathrm{mm})^{*}\end{array}$ \\
\hline Medium single & MS & $1.42(0.15)$ \\
Narrow twin & NT & $2.55(0.01)$ \\
Wide twin & WT & $4.38(0.03)$ \\
Lewis & LW & $5.16(0.01)$ \\
\hline
\end{tabular}

* Mean of measurement with standard deviation in parentheses.

were larger than the Tukey interval were statistically significant.

\section{RESULTS}

The average diameter of the 0.018 inch stainless steel arch wire was $0.440 \mathrm{~mm}(0.0174$ inches $)$. The moment of inertia (I) and modulus of elasticity (E) were $1.89 \times 10^{-3} \mathrm{~mm}^{4}\left(4.53 \times 10^{-9} \mathrm{in}^{4}\right)$ and $1.80 \times 10^{4}$ $\mathrm{kg} / \mathrm{mm}^{2}\left(25.9 \times 10^{6} \mathrm{Ib} / \mathrm{in}^{2}\right)$, respectively. Intrabracket dimensions are listed in Table I. The Lewis bracket had the greatest width at $5.16 \mathrm{~mm}$ and the medium single bracket was smallest at $1.42 \mathrm{~mm}$.

The average values of $\mathrm{N}$ with standard deviations in parentheses were $48.6(0.9)$ for two-bracket samples with free-end supports and $189.1(1.5)$ for two-bracket 
Table II. $\mathrm{N}$ values for wire under conditions of $\mathrm{O}$-ring and metal ligatures, two lengths, and two or four anchor brackets

\begin{tabular}{c|c|c|c|c}
\hline & \multicolumn{4}{|c}{ Type of bracket } \\
\cline { 2 - 5 } Conditions & Medium single (MS)* & Narrow twin (NT)* & Wide twin $($ WT)* & Lewis (LW)* \\
\hline O-ring, $\mathrm{L}_{1}, 2$ anchor brackets $\left(\mathrm{E}, \mathrm{L}_{1}, 2 \mathrm{~b}\right)$ & $69.4(0.5)$ & $79.1(2.3)$ & $115.6(1.1)$ & $94.6(1.2)$ \\
O-ring, $\mathrm{L}_{2}, 2$ anchor brackets $\left(\mathrm{E}, \mathrm{L}_{2}, 2 \mathrm{~b}\right)$ & $77.2(0.5)$ & $88.5(1.6)$ & $135.2(0.4)$ & $103.7(2.8)$ \\
O-ring, $\mathrm{L}_{1}, 4$ anchor brackets $\left(\mathrm{E}, \mathrm{L}_{1}, 4 \mathrm{~b}\right)$ & $110.4(0.8)$ & $117.2(0.3)$ & $126.4(0.5)$ & $114.8(0.7)$ \\
O-ring, $\mathrm{L}_{2}, 4$ anchor brackets $\left(\mathrm{E}, \mathrm{L}_{2}, 4 \mathrm{~b}\right)$ & $125.4(1.6)$ & $131.3(1.0)$ & $143.5(0.8)$ & $132.1(1.0)$ \\
Metal, $\mathrm{L}_{1}, 2$ anchor brackets $\left(\mathrm{M}, \mathrm{L}_{1}, 2 \mathrm{~b}\right)$ & $138.8(0.4)$ & $144.7(0.2)$ & $137.0(1.0)$ & $146.4(0.3)$ \\
Metal, $\mathrm{L}_{2}, 2$ anchor brackets $\left(\mathrm{M}, \mathrm{L}_{2}, 2 \mathrm{~b}\right)$ & $144.9(0.7)$ & $161.3(2.2)$ & $143.7(2.1)$ & $162.8(0.9)$ \\
Metal, $\mathrm{L}_{1}, 4$ anchor brackets $\left(\mathrm{M}, \mathrm{L}_{1}, 4 \mathrm{~b}\right)$ & $138.6(1.5)$ & $144.0(1.4)$ & $137.3(0.3)$ & $147.5(0.7)$ \\
Metal, $\mathrm{L}_{2}, 4$ anchor brackets $\left(\mathrm{M}, \mathrm{L}_{2}, 4 \mathrm{~b}\right)$ & $143.5(0.5)$ & $161.0(0.7)$ & $143.5(2.1)$ & $162.8(0.5)$ \\
\hline
\end{tabular}

* Means of three replications with standard deviations in parentheses. Value of Tukey interval for comparing differences among types of brackets was 0.93 . Value of Tukey interval for comparing differences among other variables was 0.50 .

samples with rigidly fixed (no rotation or longitudinal movement) end supports. Theoretic values of $\mathrm{N}$ for these same situations are 48 and 192 , respectively.

Table II lists the means and standard deviations of $\mathrm{N}$ for all combinations of variables. Fig. $5(A$ and $B)$ shows the results graphically. The value of $\mathrm{N}$ for samples with metal ties was always greater than, or in one case equal to, $\mathrm{N}$ for samples with $\mathrm{O}$-ring ties. Only in the wide twin four-bracket sample at $\mathrm{L}_{2}$ (WT, $4 \mathrm{~b}, \mathrm{~L}_{2}$ ) was there no significant difference between $\mathrm{O}$-ring and metal ties $(\mathrm{N}=143.5)$. $\mathrm{N}$ ranged from a minimum of 69.4 (MS, E, $\left.\mathrm{L}_{1}, 2 \mathrm{~b}\right)$ to a maximum of $162.8(\mathrm{LW}, \mathrm{M}$, $\mathrm{L}_{2}, 4 \mathrm{~b}$ ).

It was found that both the number of anchor brackets in the test segment and the interbracket distance had significant influence on $\mathrm{N}$. In four-bracket anchor segments with $\mathrm{O}$-ring ties, the value of $\mathrm{N}$ was always greater than in two-bracket segments with O-ring ties. Values of $\mathrm{N}$ for an interbracket distance of $\mathrm{L}_{2}$ were always greater than those at $L_{1}$, regardless of the type of ligature tie used.

A number of interactions existed for the various anchor brackets and the ligature ties that secured the arch wires to them. Values of $\mathrm{N}$ for samples of wide twin brackets with O-ring ties were always greater than $\mathrm{N}$ for samples of all other types of brackets with O-ring ties. Values of $N$ for samples of medium single brackets with O-ring ties were always less than $\mathrm{N}$ for samples of all other types of brackets with O-ring ties. In twobracket samples with $\mathrm{O}$-ring ties, $\mathrm{N}$ values were always greater with the Lewis bracket than with the narrow twin bracket. There was no significant difference found between the Lewis and narrow twin brackets in fourbracket samples with $\mathrm{O}$-ring ties.

Values of $\mathrm{N}$ for samples of Lewis and narrow twin brackets with metal ties were similar and always greater than $\mathrm{N}$ for wide twin and medium single brackets. Values of $\mathrm{N}$ for the wide twin and medium single brackets were very similar.

\section{DISCUSSION}

If information gathered with the aid of an experimental model is to be of value, the model must adequately simulate a real situation. To determine the validity of the model used in this study, it was necessary to compare experimental $\mathrm{N}$ values with ones predicted theoretically. The samples with ends free to pivot $(\mathrm{N}=48.6)$ and ends rigidly fixed $(\mathrm{N}=189.1)$ simulated an ideal beam in which theoretic $N$ values were 48 and 192, respectively. These differences are less than $1.5 \%$ and support the validity of the experimental model for testing deflection of beams. Because any small segment of an orthodontic appliance may be considered a beam (the arch wire) with supports (the anchor brackets), ${ }^{1,2}$ the model could be of value for studying configuration of simple appliances.

The force system generated by an orthodontic appliance is dependent upon appliance configuration, cross-sectional size and shape of the arch wire, and material properties of the arch wire.$^{6-9} \mathrm{~A}$ change in any of these factors may alter $\mathrm{N}, \mathrm{E}, \mathrm{I}$, or $\mathrm{L}$ of Equation 1 , thereby affecting $F$ for a given $D$. Certain configurational chariges were imposed upon the original beam model and their effects analyzed. All changes basically altered the stiffness of the beam as anchored to the supports. The value of $\mathrm{N}$ reflects stiffness of the supports.

$\mathrm{N}$ is directly related to $\mathrm{F}$ (force or load) as described by Equation 1 . Clinically, the force can be thought of as a reactive force stored within the arch wire after activation, which causes tooth movement as it is dissipated. The orthodontist must apply a load to the arch 


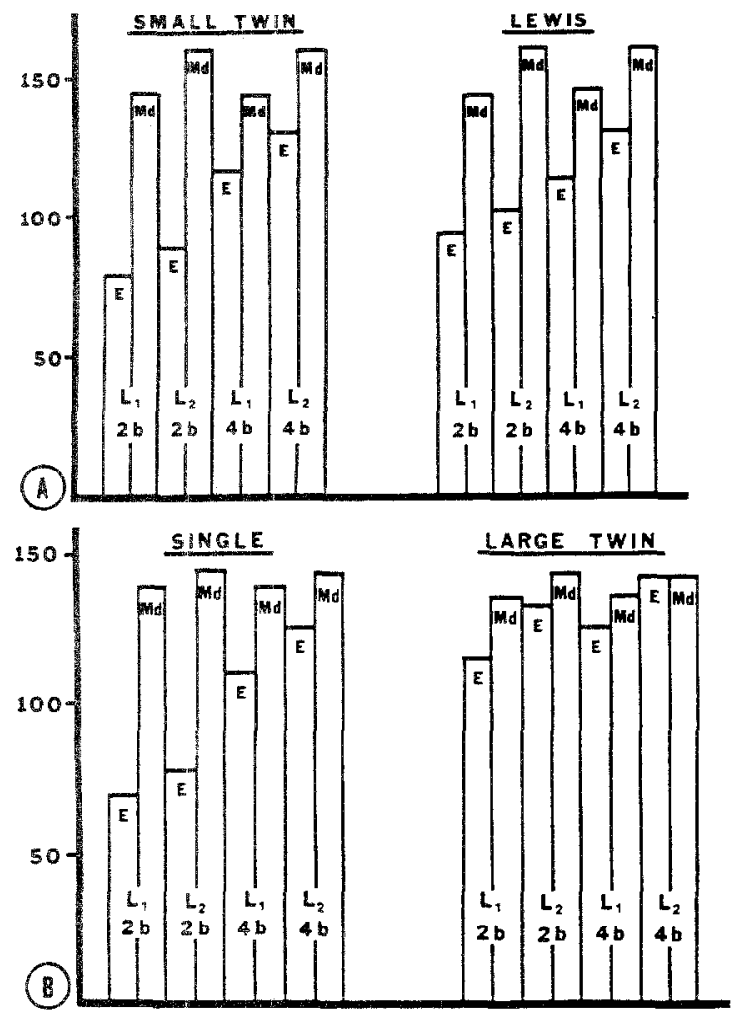

Fig. 5. The effect of elastic (E) and metal (M) ties on $N$ for all combinations of brackets, interbracket distances $\left(L_{1}\right.$ vs. $\left.L_{2}\right)$, and number of anchor brackets ( $2 \mathrm{~b}$ vs. $4 \mathrm{~b}$ ). A, Narrow twin (left) and Lewis (right). B, Medium single (left) and wide twin (right).

wire to engage it into the bracket slots. The position of the bracket slots is determined by the three-dimensional configuration of the malocclusion. The load may be in the form of a twist or deflection or both, depending on the spatial arrangement of the bracket slots and crosssectional shape of the arch wire (round or rectangular). It is the reactive force of the elastically twisted or deflected arch wire that is responsible for tooth movement. The magnitude of this force varies in direct proportion to changes in $\mathrm{N}$.

The value of $\mathrm{N}$ for samples with metal ties was greater than, or in one instance equal to, $\mathrm{N}$ for samples with O-ring ties because the metal ligature ties secured the arch wire more rigidly to the brackets than did $\mathrm{O}-$ rings. Therefore, in a clinical situation with conditions similar to those simulated by the model, a fully engaged arch wire tied in with metal ties is likely to exert more reactive force on the teeth than one tied in with 0 -rings.

The wider the bracket, the more an O-ring must be stretched to accommodate that bracket. Because $\bigcirc$-rings become more rigid when stretched, the rigidity with which the arch wire is fastened to the brackets is increased. It was not surprising to find that with o-ring
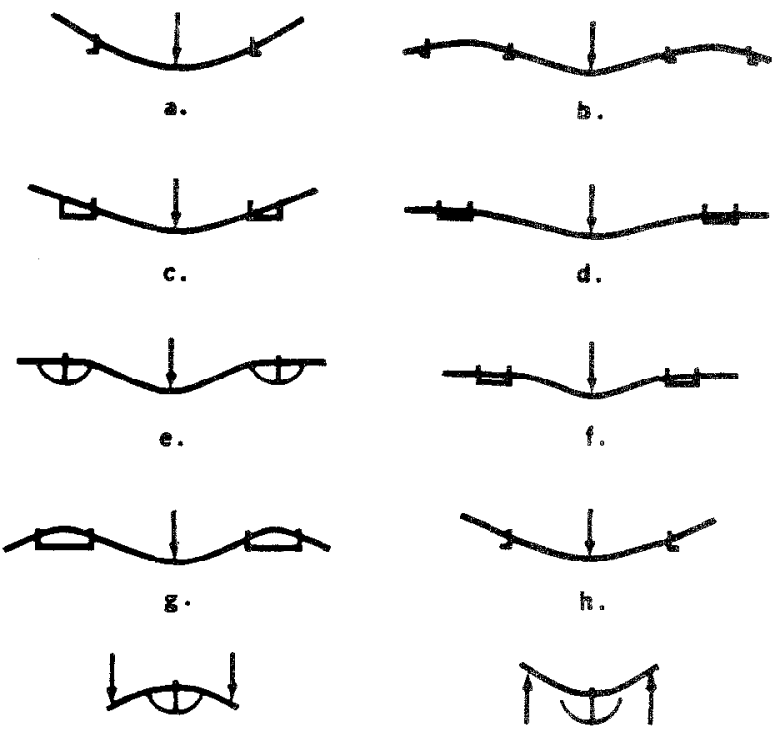

i.

.

Fig. 6. The stiffness of various bracket arrangements. $a$, Lack of stiffness in two-bracket samples with o-ring ties. $b$, Increased stiffness associated with the addition of two extra anchor brackets. $c$, Decreased stiffness caused by stretching of the ligature ties at $L_{1}$. $d$, Less stretch and increased stiffness at $L_{2}$. e, The most rigid arrangement-Lewis brackets with metal ties. $f_{s}$ Also very rigid, narrow twin brackets with metal ties. $g$, Intrabracket bowing decreases stiffness with wide twin brackets. $h$, Decreased stiffness associated with the narrow intrabracket width of medium single brackets. $i$, Lingual forces activate Lewis wings. $j$, Lewis wings are not activated with buccal forces.

ties $\mathrm{N}$ was greatest for the widest brackets. In fact, for most wide twin brackets $\left(4 b, L_{2}\right)$, there was no difference between the reactive forces generated with elastic ties and those generated with metal ties. In contrast, the lowest forces were associated with medium single brackets (the narrowest bracket) and O-ring ties.

In samples with O-ring ties, $\mathrm{N}$ was greater for the larger four-bracket segments than for the smaller twobracket segments. Fig. $6, a$, illustrates the lack of rigidity inherent with $\bigcirc$-ring ties, which allowed a certain amount of pivoting to occur at the supports in twobracket samples. The amount of pivot was reduced by the two additional brackets in a four-bracket segment (Fig. 6, b). This demonstrates the influence that components removed from the immediate site of activation can have on the forces produced at that site. Because more components were incorporated into the larger (four-bracket) samples, they were considered more representative of the clinical situation than two-bracket samples. The forces produced from four-bracket segments of Lewis and small twin brackets were very similar. Perhaps this same relationship exists in the mouth.

Samples with the greater interbracket distance $\left(\mathrm{L}_{2}\right)$ 
consistently produced greater values of $\mathrm{N}$ regardless of the type of brackets, type of ligature ties, or size of the test segment. This is curious because theoretically variation of $\mathrm{L}$ should not affect $\mathrm{N}$. One possible explanation is that at a shorter interbracket distance $\left(\mathrm{L}_{1}\right)$, a greater load is required to deflect the arch wire a given amount than at the longer distance $\left(L_{2}\right)$. An increased load at the center of the arch wire creates higher forces at the brackets and specifically on the ligature ties. Fig. 6, $c$ and $d$, illustrates how the higher forces at $\mathrm{L}_{1}$ may cause more arch wire movement at the support brackets with stretching and distortion of the ligature ties. Ligature ties appear to be less rigid at shorter interbracket distances than at longer ones. Orthodontists occasionally select brackets that are narrow mesiodistally to increase the interbracket distance. This is appealing if lighter forces and greater amounts of activation are desired. If, however, $\mathrm{N}$ increases as $\mathrm{L}$ increases, the reduction in force created by increased interbracket distances may not be as substantial as would be expected if $\mathrm{N}$ remained the same for $L_{1}$ and $L_{2}$.

Samples with metal ties and Lewis or small twin brackets were very similar and consistently produced the highest $\mathrm{N}$ values. Fig. 6 , $e$, illustrates how the unique design of the Lewis bracket encourages a very rigid support when tied with metal ties. The three-point contact permits very little pivot or bowing of a lingually deflected arch wire either at or within the bracket. Narrow twin brackets apparently are large enough to discourage excessive pivoting of the arch wire at the bracket's edge, yet small enough to minimize the amount of bowing of the arch wire within the bracket itself (Fig. 6, $f$ ).

Samples with metal ties and wide twin or medium single brackets were similar. They consistently produced the lowest $N$ values of all samples with metal ties. It is speculated that the great intrabracket width of large twin brackets allows the arch wire to bow within the bracket as illustrated in Fig. $6, g$. The bowing causes a decrease in rigidity that is reflected in smaller $\mathrm{N}$ values. Medium single brackets are very narrow and allow the arch wire to pivot on the inner edge (Fig. 6, $h$. This results in loss in rigidity and correspondingly small $\mathrm{N}$ values.

The relationships among types of brackets, regardless of the ligature ties used, become more complicated when applied clinically. For example, twin brackets are often used in certain segments of the dental arch because they offer the best control over both rotation and mesiodistal axial inclination. There is general recognition of the fact that twin brackets tend to decrease interbracket distance $(\mathrm{L})$ and thereby increase the reactive force of the arch wire in that region. What is not so obvious, however, is that the force increase caused by a smaller interbracket width may be less than expected with wide twin brackets and/or more than expected with narrow twin brackets because of variation in $N$ values. On the other hand, the smaller medium single brackets are used in situations in which lighter forces are desired, thereby taking advantage of the greater interbracket distance. Because of their unique design (Fig. 6, $i$ and $j$ ), the Lewis brackets may be thought to offer the increased interbracket width of medium single brackets without sacrificing the control of twin brackets. It must be remembered, however, that lingual and rotational movements will activate the Lewis wings and much heavier than expected forces could be produced.

This study demonstrates the complexity of the interactions that take place with an orthodontic appliance. With simulated tests, it is possible to identify and quantify unknowns such as $\mathrm{N}$. This brings us one step closer to an accurate theoretic and mathematic description of the orthodontic force system.

With the use of a model, it is possible to evaluate the performance of materials in service. It must, however, be understood that the test model in this study simulated configurations of very simple appliances. The results are believed to reflect general trends for what may occur clinically in situations similar to those simulated. Clinical application of these findings to more complex arrangements may be misleading.

\section{REFERENCES}

1. Jarabak JR. Technique and treatment with the light wire appliance. 2nd ed. St. Louis: The C. V. Mosby Company, 1963.

2. Thurow RC. Edgewise orthodontics. 4 th ed. St. Louis: The C. V. Mosby Company, 1982.

3. Graber TM. Orthodontics, principles and practice. 2 nd ed. Philadelphia: WB Saunders, 1972.

4. Dalby $\mathbf{J}$ (programmer). BMD8V-Analysis of variance. Ann Arbor: Statistical Research Laboratory, University of Michigan, 1968.

5. Guenther WC. Analysis of variance. Fnglewood Cliffs, NI: Prentice-Hall, 1964:141.

6. Burstone $\mathrm{CJ}$, Goldberg AJ. Maximum forces and deflections from orthodontic appliances. AM J ORTHOD 1983;84:95-103.

7. Creekmore TD. The importance of interbracket width in orthodontic tooth movement. J Clin Orthod 1976;10:530-4.

8. Burstone CJ. The application of continuous forces to orthodontics. Angle Orthod 1961;31:1-14.

9. Burstone CJ. The rationalc of the segmented arch. AM J ORTHOD 1962;11:805-21:

Request reprints to:

Dr. John M. Powers

Department of Biomaterials

University of Michigan

School of Dentistry

Ann Arbor, MI 48109-1078 